\title{
INNOVACIÓN DOCENTE A LA LUZ DE BOLONIA: TRABAJO EN EQUIPO Y REVISIONES CRUZADAS PARA CONVERTIR AL ALUMNO EN PROTA- GONISTA DE SU PROCESO DE APRENDIZAJE
}

\author{
María del Rosario González Martín y Gonzalo Génova Fuster \\ marrgonz@edu.ucm.es \\ ggenova@inf.uc3m.es
} Facultad de Educación. Universidad Complutense de Madrid y Escuela Politécnica Superior. Universidad Carlos

Resumen: A la luz del Proceso de Bolonia hemos realizado una adaptación del método de enseñanza de una asignatura de la titulación de Ingeniería Informática, basado en la adquisición de competencias genéricas y transversales, en el protagonismo del alumno y en el trabajo en equipo. Del mismo modo se ha adaptado el procedimiento de evaluación al nuevo modelo de enseñanza. Después de tres años de implantación y mejora, el resultado ha sido satisfactorio en relación a objetivos y competencias, tanto para alumnos como para profesores.

Palabras clave: innovación docente, trabajo en equipo, competencias genéricas y transversales, protagonismo del alumno. 


\section{INNOVATION TEACHING IN THE LIGHT OF BOLOGNA: WORKGROUP AND CROSS REVISIONS TO CONVERT THE STUDENT IN PROTAGONIST OF ITS LEARNING PROCESS}

Abstract. Under the light of the Bologna Process, we have accomplished an adaptation of the learning method of a course belonging to the Computer Engineering degree, based on the acquisition of generic and transversal competences, on the student's protagonism, and on team work. Similarly, we have adapted the evaluation process to the new learning model. After three years of implantation and improvement, the result has been satisfactory regarding goals and competencies, both for students and for lecturers.

Key words: teaching innovation, team work, generic and transversal competences, student's protagonism.

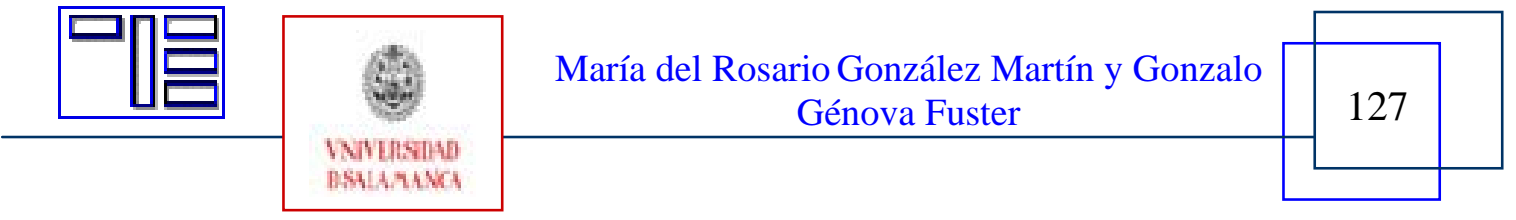


À la lumière du processus de Bologne, nous avons effectué des ajustements de la méthode d'enseignement d'un sujet sur le degré de Computer Engineering, basée sur l'acquisition de compétences transversales et génériques, dans le rôle de l'élève et le travail d'équipe. De même, a adapté la procédure d'évaluation pour le nouveau modèle d' enseignement. Après trois années de mise en œuvre et l'amélioration, le résultat a été satisfaisant en ce qui concerne les objectifs et les compétences, à la fois pour les élèves et pour les enseignants.

Mots clés: innovation pédagogique, travail d'équipe, compétences génériques et propriété croisée de l'élève. 


\begin{tabular}{|c|c|c|}
\hline \multirow[t]{2}{*}{ ロ包 } & \multicolumn{2}{|c|}{$\begin{array}{l}\text { Revista Electrónica Teoría de la Educación. } \\
\text { Educación y Cultura en la Sociedad de la Información. }\end{array}$} \\
\hline & http://www.usal.es/teoriaeducacion & Vol. 9. No 1. Febrero 2008 \\
\hline
\end{tabular}

\title{
INNOVACIÓN DOCENTE A LA LUZ DE BOLONIA: TRABAJO EN EQUIPO Y REVISIONES CRUZADAS PARA CONVERTIR AL ALUMNO EN PROTA- GONISTA DE SU PROCESO DE APRENDIZAJE
}

\author{
María del Rosario Gonzalez Martín y Gonzalo Génova Fuster \\ marrgonz@edu.ucm.es \\ ggenova@inf.uc3m.es \\ Facultad de Educación. Universidad Complutense de Madrid y Escuela Politécnica Su- \\ perior. Universidad Carlos
}

\section{1.- INTRODUCCIÓN}

La innovación docente es una exigencia que apela a todo docente que se preocupa por una enseñanza activa en diálogo con las necesidades fundamentales y variables del alumnado y de la sociedad para la que se prepara. Si esto es así en toda circunstancia, lo es aún más en el marco del Proceso de Bolonia. Una de las consignas principales, entre otras, de la didáctica de este proceso es el protagonismo del alumno en su propia formación. Él ha de ser el responsable principal de su formación y el sujeto más activo en la misma. Para que el alumno asuma este protagonismo también necesita de la motivación del docente y, por supuesto, de que éste adapte su forma de enseñar. No todos los modos de enseñar fomentan un modo activo de aprender. Con esta experiencia de innovación docente hemos querido comenzar a devolver al alumno el protagonismo de su propia formación. Al mismo tiempo, hemos querido contar con las exigencias formativas de nuestra sociedad y por ello hemos creído conveniente que este protagonismo activo de la educación no sólo sea desde una perspectiva individual sino también grupal, ya que la mayoría de trabajos (de implementación de proyectos, de valoraciones de los mismos, etc.) se realizan en equipo. Los alumnos deben acostumbrarse a crear en equipo, a implementar de manera coordinada, a valorar a sus colegas y a ser valorados por ellos, a recibir críticas de compañeros y encajarlas de forma óptima y no defensiva, a proponer criterios de evaluación... En definitiva, con nuestro trabajo hemos querido realizar un diseño didáctico de innovación docente en el que los principales requisitos son dos: que el alumno asuma el protagonismo de su propia formación (siguiendo las recomendaciones del proceso de Bolonia) y que aprenda a trabajar en equipo, desarrollando actitudes y habilidades de comunicación que serán necesarias en el día a día de su trabajo futuro, porque lo requiere la sociedad laboral actual.

Este artículo presenta el Proyecto de Innovación Docente llevado a cabo durante el curso 2004-2005 en el ámbito de la asignatura Ingeniería del Software II, de la titulación Ingeniería en Informática, en la Escuela Politécnica Superior de la Universidad Carlos III de Madrid. La metodología iniciada en este Proyecto ha sido continuada y mejorada desde entonces en los sucesivos cursos académicos, y aplicada así mismo a la asignatura "hermana” Ingeniería del Software I. Ambas asignaturas son troncales de 6 créditos, y se imparten respectivamente en el primer y segundo cuatrimestre del cuarto curso de la titulación.

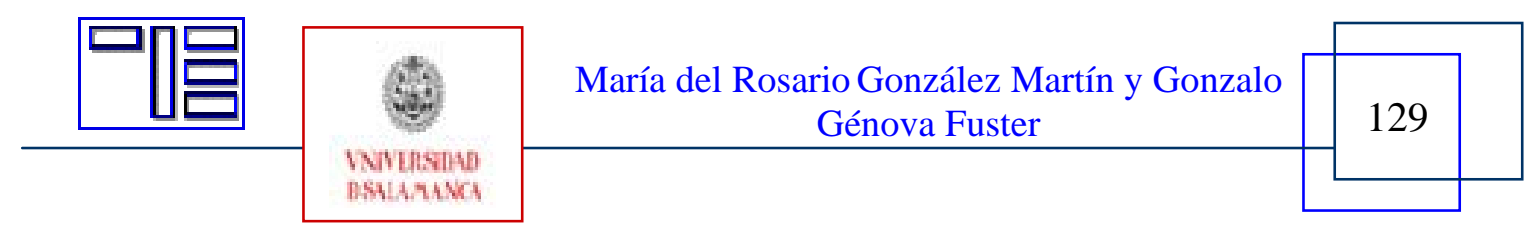


El núcleo de este proyecto consiste en fomentar el trabajo en equipo de los alumnos supervisado por los profesores de la asignatura, junto con las revisiones cruzadas que unos equipos realizan al trabajo de otros. Con estas revisiones cruzadas se pretende que los alumnos aprendan de los errores que detectan en los trabajos de sus compañeros, ya que, al verse obligados a revisar rigurosamente esos trabajos ajenos, identifican en ellos los errores más fácilmente que los suyos propios. Al recibir la corrección de sus propios compañeros, también se dan cuenta de que sus defectos no responden a juicios caprichosos de los profesores. El trabajo en equipo, el recibir críticas de los compañeros, el revisar su proyecto a la luz de esas críticas, la presentación en público de sus trabajos de equipo, etc., les prepara para una actividad y les entrena en unas actitudes que tendrán que desarrollar sin duda en su vida profesional. En definitiva, se trabaja el desarrollo de competencias tanto genéricas como trasversales en el marco de las propuesta333333s para la renovación de las metodologías educativas en la Universidad.

Aunque la descripción del proyecto de innovación se centra en una asignatura concreta $y$, en consecuencia, el modo de desarrollarlo se adapta a los requerimientos de dicha asignatura, la didáctica concreta que proponemos puede aplicarse a aprendizajes diversos. No obstante, y puesto que no hemos querido perder rigor en la presentación de la experiencia, algunas descripciones pueden resultar demasiado precisas para un lector que quiera aplicar este modo de innovación a otras áreas. En las secciones 2, 3 y 4 se describen los objetivos, método de trabajo y resultados alcanzados en el Proyecto de Innovación Docente, mientras que la sección 5 presentamos nuestras conclusiones y propuestas de aplicación a otros ámbitos de conocimiento.

\section{2.- OBJETIVOS}

La documentación de proyectos es una labor esencial en la Ingeniería Software, y por supuesto en casi todas las áreas de conocimiento: imprescindible para razonar y comunicar adecuadamente el alcance y desarrollo del proyecto en todas sus fases. Es importante tanto la corrección formal (adecuación del documento a las normas previstas en un estándar de documentación), como, obviamente, la corrección material (contenido bien razonado, desarrollado de modo coherente, ordenado y completo): ambas constituyen en buena medida la garantía del éxito de un proyecto. Por otra parte, los alumnos deben aprender también a realizar los trabajos en equipo, a presentar y justificar sus trabajos ante otras personas, y a revisar y corregir trabajos ajenos.

La Tabla 1 contiene un resumen de los objetivos detallados de este proyecto de innovación, y de los indicadores empleados para evaluar los resultados alcanzados en cada objetivo. Como puede observarse, estos objetivos son perfectamente trasladables a otras áreas de conocimiento.

\begin{tabular}{|c|c|}
\hline Objetivos detallados & Indicadores de resultados \\
\hline \multirow{2}{*}{$\begin{array}{l}\text { Conocimiento y aplicación } \\
\text { de un estándar de documen- } \\
\text { tación de proyectos infor- } \\
\text { máticos }\end{array}$} & $\begin{array}{l}\text { Adecuación de los trabajos presentados al estándar de } \\
\text { documentación }\end{array}$ \\
\hline & $\begin{array}{l}\text { Adecuación de las revisiones de trabajos ajenos al mismo } \\
\text { estándar }\end{array}$ \\
\hline
\end{tabular}

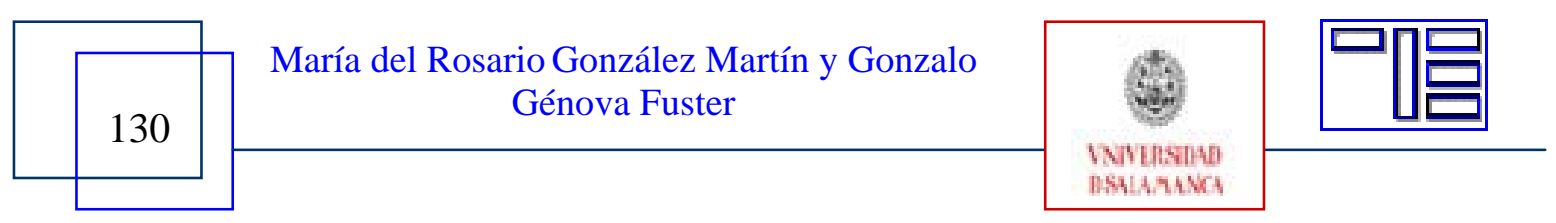




\begin{tabular}{|l|l|}
\hline \multirow{3}{*}{ Trabajo en equipo } & Evaluación global del trabajo del equipo \\
\cline { 2 - 3 } Presentaciones en público & $\begin{array}{l}\text { Evaluación individual de la tarea específica de cada } \\
\text { miembro del equipo }\end{array}$ \\
\hline & $\begin{array}{l}\text { Evaluación de la calidad de la presentación: medios au- } \\
\text { diovisuales, expresión oral, lenguaje corporal }\end{array}$ \\
\cline { 2 - 2 } & $\begin{array}{l}\text { Evaluación de la capacidad de respuesta a preguntas de } \\
\text { otros compañeros para justificar el trabajo presentado, } \\
\text { argumentando de modo racional }\end{array}$ \\
\cline { 2 - 2 } & $\begin{array}{l}\text { Evaluación de la capacidad de evaluar el trabajo de los } \\
\text { compañeros de modo objetivo y respetuoso, y de asumir } \\
\text { las revisiones recibidas }\end{array}$ \\
\hline
\end{tabular}

Tabla 1. Objetivos detallados e indicadores de resultados

\section{3.- MÉTODO DE TRABAJO}

\subsection{Metodología docente}

Las dos asignaturas constan principalmente de cuatro bloques teóricos: captura de requisitos, análisis, diseño de alto nivel, diseño de bajo nivel. La práctica se desarrolla en equipos de cuatro alumnos, en cuatro fases correspondientes a los cuatro bloques teóricos. En cada fase se deben realizar tres tareas principales:

Desarrollo razonado del proyecto, que debe apoyarse en la documentación adecuada de acuerdo con el estándar de ingeniería del software explicado en las clases teóricas (PSS05-0 de la Agencia Espacial Europea). El resultado son cuatro documentos distintos: URD, SRD, ADD y DDD (las siglas en inglés corresponden a Documento de Requisitos del Usuario, Documento de Requisitos del Software, Documento de Diseño Arquitectónico, y Documento de Diseño Detallado).

Tutorías por equipo. Cada equipo se reúne con un profesor de la asignatura para exponer el trabajo en curso y resolver posibles problemas. Los alumnos deben acudir con borradores o esquemas para poder formular preguntas concretas. Las tutorías se asemejan a sesiones de consultoría, en las que unos ingenieros inexpertos preguntan a otros ingenieros más expertos.

Revisión del proyecto presentado por otro equipo. Cada equipo entrega la documentación de cada fase a otro equipo designado por los profesores. El equipo revisor debe revisar la forma y contenido de la documentación entregada, y debe emitir un breve informe escrito con el contenido de la revisión, que será entregado al equipo autor original. El equipo revisado deberá actualizar el documento del proyecto de acuerdo con la revisión recibida del equipo revisor. Es una ocasión excelente para aprender a corregir, aprender a ser corregido, y aprender de los errores propios y ajenos, en el contexto de valores como la crítica, el razonamiento, la defensa, la moderación, el respeto, etc. Presentación en público con medios audiovisuales durante las clases de prácticas. El público asistente (toda la clase, y en particular el equipo revisor) puede formular las

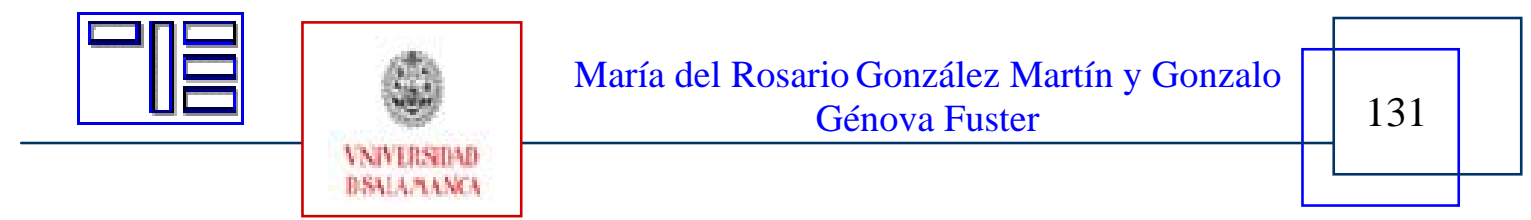


preguntas que considere oportunas, y el ponente debe defender de modo razonado el proyecto que presenta. El profesor modera la sesión, y aprovecha para destacar las nociones teóricas relevantes. Es una ocasión excelente para fomentar la participación de todos los alumnos. Todos los alumnos deberán realizar al menos una presentación a lo largo del curso, lo que garantiza también el compromiso de cada alumno con el trabajo del equipo.

Esta metodología requiere una planificación muy cuidadosa del curso, en la que deben estar claros desde el principio los procedimientos y fechas de entrega de cada fase de la práctica.

\subsection{Sistema de evaluación}

La calificación final de la asignatura, resumida en la Tabla 2, tiene una parte práctica y una parte teórica, y a la vez cada elemento de calificación puede ser común a todo el equipo (60\%) o individual de cada alumno (40\%). En la parte práctica (70\%) se evalúan los documentos presentados en su versión final revisada (corrección formal y material), las presentaciones en público realizadas (preparación y ejecución), y los informes de revisión sobre los trabajos de otros equipos. Para facilitar que los alumnos acepten que su revisión en ningún caso es un perjuicio para sus compañeros, sino más bien una ayuda para mejorar, la revisión no influye en la calificación de la práctica del otro equipo. Para la parte teórica (30\%), al final del curso cada equipo de prácticas debe elaborar una propuesta de preguntas de test para el examen final de la asignatura. Cada equipo debe proponer tres preguntas de test de respuesta múltiple, con sus correspondientes respuestas, señalando la que consideren válida. La propuesta es evaluada por los profesores en cuanto a su corrección y su capacidad discriminatoria de conocimientos adquiridos; se valora especialmente que el equipo haya identificado nociones clave del contenido teórico de la asignatura y formule preguntas adecuadas acerca de ellas. Posteriormente los profesores realizan una selección de preguntas de test a partir de las propuestas, y con ellas confeccionan el examen teórico final. La parte teórica de la calificación contempla ambos aspectos.

\begin{tabular}{|l|l|l|l|}
\hline \multirow{3}{*}{$\begin{array}{l}70 \% \\
\text { Práctica }\end{array}$} & $40 \%$ & $\begin{array}{l}\text { Documentación presen- } \\
\text { tada }\end{array}$ & Equipo \\
\cline { 2 - 4 } & $20 \%$ & Exposición pública & Individual \\
\cline { 2 - 4 } & $10 \%$ & Informes de revisión & Equipo \\
\hline $30 \%$ & $10 \%$ & Propuesta de preguntas & Equipo \\
\cline { 2 - 4 } Teórica & $20 \%$ & Examen tipo test & Individual \\
\hline
\end{tabular}

Tabla 2. Esquema de calificación de la asignatura

\subsection{Prácticas requeridas a los alumnos}

En el curso académico 2004-2005 la práctica tuvo el siguiente enunciado, que pretendimos fuera lo más sencillo posible:

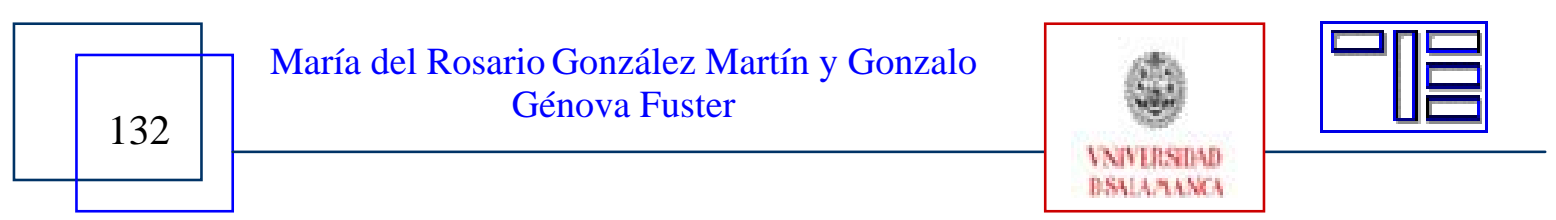


"Necesito algo para organizar mejor mis actividades, una agenda para llevar al día mi horario, mis compromisos, etc."

No teléfonos, ni contactos... sólo organización de calendario.

Libertad para escoger plataforma e interacción con el usuario

Extensión de los documentos (orientativa): URD, 5 a 10 páginas; SRD, ADD y DDD, 10 a 20 páginas cada uno; total, 35 a 70 páginas. Se hizo hincapié en que interesa la calidad, no la cantidad, de los documentos presentados.

Los alumnos se constituyeron en un total de 28 equipos de trabajo, con cuatro componentes por equipo, con las necesarias excepciones. En cursos sucesivos se pidieron prácticas semejantes a la agenda electrónica, tales como una aplicación de contabilidad doméstica, un sistema de control centralizado y flexible de electrodomésticos, una cocina robotizada, etc.

\section{4.- RESULTADOS ALCANZADOS}

\subsection{Volumen de trabajo}

La realización de la práctica supuso el volumen de trabajo reflejado en la Tabla 3, en promedio por equipo, medido en páginas del trabajo presentado y horas dedicadas a su realización en cada una de sus fases, según datos facilitados por los propios alumnos.

\begin{tabular}{|l|l|l|l|l|l|l|l|}
\hline Documento & URD & SRD & ADD & DDD & Unificación & Exposición & Total \\
\hline Páginas & 16,2 & 38,5 & 35,4 & 34,6 & 72,3 & 20,8 & 217,8 \\
\hline Horas & 60,2 & 85,1 & 79,8 & 67,8 & 22,0 & 14,1 & 329,1 \\
\hline
\end{tabular}

Tabla 3. Esfuerzo requerido para la realización de la práctica en cada una de sus fases

El tamaño final de la práctica osciló entre 140 y 252 páginas, y la dedicación entre 162 y 537 horas por equipo: la dispersión es muy grande, y pueden influir métodos poco objetivos de contar las horas de dedicación a la práctica por parte de los propios alumnos. El ratio resultante es de 1,5 horas/página.

Nótese que la extensión efectiva de los documentos presentados es aproximadamente el triple que los límites orientativos que se les dieron. Probablemente estos límites eran excesivamente optimistas, y no era realmente posible ajustarse a ellos y seguir a la vez el estándar de documentación de la asignatura, pero en todo caso la impresión de los profesores es que los alumnos se excedieron en el trabajo, muy por encima de lo exigido en la asignatura.

La dedicación a la práctica supone unas 82 horas de trabajo por alumno, que equivale a unos 3 créditos ECTS. A esto hay que sumar las horas lectivas (60 horas), las horas dedicadas a preparar la propuesta de preguntas de examen (valor desconocido, probablemente en torno a 3-5 horas), el tiempo de realización del examen (1 hora), y tal vez algunas pocas horas más dedicadas a actividades sin determinar bien. En total, no parece que el tiempo dedicado a la asignatura haya sido desproporcionado. Esto contrasta con la opinión de los propios alumnos de que habían dedicado a la asignatura entre el doble y el triple de esfuerzo que a las demás asignaturas troncales del curso (véanse más abajo

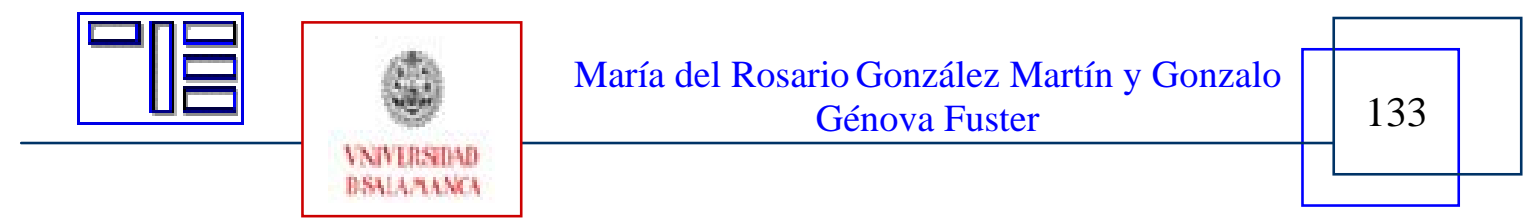


los resultados de la encuesta). Considerando también que el ratio de 1,5 horas/página es más bien escaso, tal vez sea necesario inferir que los alumnos fueron excesivamente conservadores a la hora de estimar el esfuerzo en horas que les llevó la práctica, es decir, que en realidad dedicaron más tiempo del que ellos mismos nos indican. De hecho, según los datos obtenidos por otra vía mediante la Encuesta de Evaluación de la Docencia, el valor promedio en respuesta a la pregunta "Estime el número de horas semanales, excluidas las clases, que ha dedicado a la asignatura” es de aproximadamente 8 horas/semana, es decir, unas 120 horas en 15 semanas. No podemos decir cuál de las dos estimaciones se acerca más a la verdad: la obtenida por nosotros tiene a su favor su mayor granularidad y la petición continua de información a lo largo de todo el cuatrimestre, mientras que el valor obtenido de la Encuesta de Evaluación de la Docencia se ajusta mejor a las consideraciones previas.

\subsection{Calificaciones}

Todas las prácticas merecieron buenas calificaciones a juicio de los profesores. Como referencia, las prácticas “correctas, pero no brillantes” fueron calificadas con 8,0. La calificación promedio fue 8,6. Cuatro de las prácticas merecieron la calificación máxima $(10,0)$, y tres de ellas obtuvieron sólo 7,0. En cursos sucesivos los resultados fueron semejantes.

Como era de esperar, no se aprecia correlación entre las calificaciones y el esfuerzo realizado por los alumnos: las mejores y peores prácticas están en la zona media, tanto en número de páginas como en número de horas.

\subsection{Examen teórico realizado por los alumnos}

La calificación promedio obtenida por los alumnos en el examen de test, de 20 preguntas, fue 9,5 (elevadísima), con una dispersión mínima. A juicio de los profesores, el enunciado del examen no era excesivamente fácil, por lo que hay que concluir que probablemente los alumnos difundieron entre ellos sus respectivas propuestas de preguntas. Se han propuesto algunas estrategias para intentar evitar que se produzca esta difusión de preguntas, pero no nos satisface ninguna de ellas. La misma táctica que nosotros pretendíamos poner en juego ("si una pregunta es acertada por todos, no cuenta en la calificación, porque indica que la pregunta ha sido difundida previamente”) no fue posible ponerla en práctica, porque el número de preguntas acertadas por todos fue muy grande. Probablemente, el mismo factor de hacer eliminatorio el examen tuvo un efecto negativo en este sentido: los alumnos no quisieron arriesgarse en un examen que cuenta relativamente poco en la calificación global, pero que es amenazadoramente eliminatorio. En cursos sucesivos hemos mitigado el efecto de la difusión de las propuestas de preguntas mediante la repetición de las mejores preguntas de años anteriores, además de hacer que las respuestas equivocadas puntuasen negativamente. De esta forma la calificación promedio del examen final ha ido bajando hasta 6,5 (mucho más razonable). En todo caso, consideramos que el objetivo principal de repasar y asimilar las nociones teóricas está plenamente conseguido, principalmente por la vía del trabajo para proponer

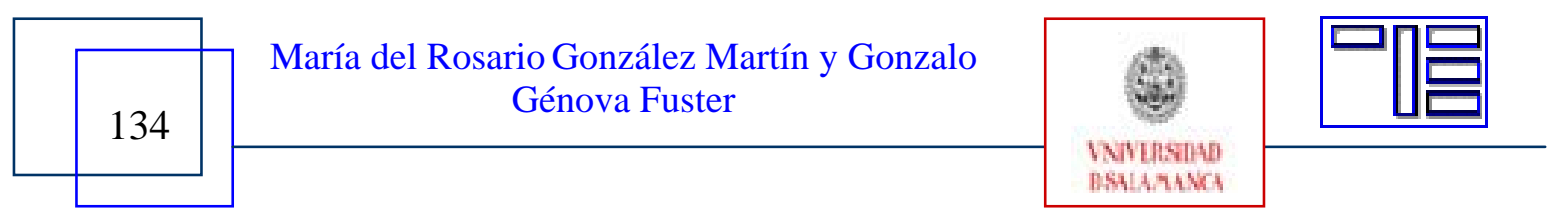


las preguntas del examen. La calificación promedio de las propuestas de preguntas fue 8,0 .

\subsection{Resultados de la encuesta de seguimiento del proyecto}

El mismo día del examen teórico se presentó a los alumnos una encuesta para que valoraran el desarrollo de la asignatura. En esta encuesta se les pedía:

- $\quad$ Valorar el grado de novedad e interés de cada uno de los temas abordados en la asignatura.

- $\quad$ Comparar el esfuerzo dedicado a la asignatura con otras asignaturas del mismo curso.

- $\quad$ Señalar algún otro tema que hubiera deseado que se viera en la asignatura.

- $\quad$ Señalar dos o tres puntos que considera son los más importantes que ha aprendido en esta asignatura (teóricos, prácticos, habilidades, capacidades, etc.).

- $\quad$ Otras sugerencias.

Realizaron el examen 95 alumnos y respondieron a la encuesta 93, de modo que la participación es elevadísima.

\subsection{Novedad e interés de la asignatura.}

Las valoraciones (ver Tabla 4) se realizaron conforme a la siguiente escala:

Novedad: 1-ya conocía perfectamente el tema; 5-era un tema completamente desconocido.

Interés: 1-no me ha interesado el tema en absoluto; 5-ha sido un tema apasionante.

\begin{tabular}{|l|l|l|}
\hline Tema & Novedad & Interés \\
\hline Estándares de documentación en el proceso de desarrollo de software & 3,84 & 2,77 \\
\hline Introducción a la ingeniería de requisitos & 2,49 & 3,24 \\
\hline Requisitos del usuario: obtención y descripción & 2,52 & 3,17 \\
\hline Requisitos del software: tipos, propiedades, organización y calidad & 2,94 & 3,23 \\
\hline Introducción al diseño arquitectónico & 3,58 & 3,55 \\
\hline Estilos arquitectónicos & 4,10 & 3,49 \\
\hline Introducción al diseño detallado & 3,35 & 3,46 \\
\hline Patrones de diseño & 2,70 & 3,51 \\
\hline Promedio & 3,19 & 3,30 \\
\hline
\end{tabular}

Tabla 4. Valoración por los alumnos del grado de novedad e interés de cada uno de los temas

El interés en general $(3,30)$ es algo superior a la novedad en general $(3,19)$, y ambos valores son algo superiores al valor "neutro" $(3,0)$. Se puede calificar como un resultado modestamente bueno. Los temas más novedosos son "Estilos arquitectónicos” y "Estándares de documentación”. Los temas menos novedosos son "Introducción a la ingeniería

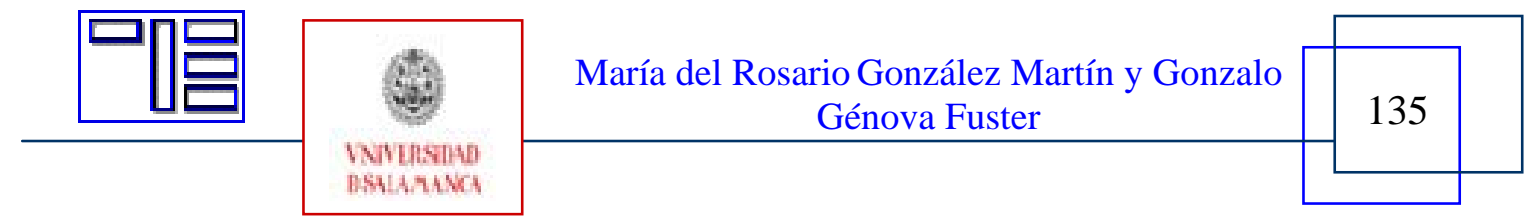


de requisitos” y "Requisitos de usuario". Los temas más interesantes son "Introducción al diseño arquitectónico" y "Patrones de diseño". Los temas menos interesantes son "Estándares de documentación” y "Requisitos de usuario”. En general, más interesantes los temas de diseño que los temas de requisitos y análisis.

\subsection{Esfuerzo dedicado a la asignatura}

Se pedía a los encuestados que comparasen en forma de cociente el esfuerzo dedicado a la asignatura objeto de estudio, "Ingeniería del Software II”, por una parte con "Ingeniería del Software I", que se imparte en el cuatrimestre precedente y constituye su base teórica, y por otra parte con el resto de asignaturas troncales del curso. Las respuestas de los alumnos fueron valoradas en la escala de 1,00 a 5,00 (ver Tabla 5). La respuesta intermedia significa "aproximadamente el mismo esfuerzo", mientras que las demás respuestas significan "más del doble”, "más del cuádruple”, "menos de la mitad” y "menos de la cuarta parte".

\begin{tabular}{|l|l|l|l|l|l|l|}
\hline $\begin{array}{l}\text { Cociente de compara- } \\
\text { ción }\end{array}$ & $\begin{array}{l}\text { Menos de } \\
0,25\end{array}$ & $\begin{array}{l}0,25 \text { a } \\
0,5\end{array}$ & $\begin{array}{l}0,5 \text { a } \\
2\end{array}$ & $\begin{array}{l}2 \text { a } \\
4\end{array}$ & $\begin{array}{l}\text { Más de } \\
4\end{array}$ & Resultado \\
\hline Ing. Soft. 2 / Ing. Soft. 1 & 1,00 & 2,00 & 3,00 & 4,00 & 5,00 & 4,34 \\
\hline $\begin{array}{l}\text { Ing. Soft. 2 / Resto de } \\
\text { TR }\end{array}$ & 1,00 & 2,00 & 3,00 & 4,00 & 5,00 & 3,78 \\
\hline
\end{tabular}

Tabla 5: Valoración numérica asignada al cociente de esfuerzos y resultado obtenido

Si el valor 4,00 significa "bastante más esfuerzo" (entre el doble y el cuádruple), y el valor 5,00 significa "mucho más esfuerzo" (más del cuádruple), el promedio global indica que los alumnos consideran que han dedicado a "Ingeniería del Software II" un esfuerzo considerablemente superior a "Ingeniería del Software I", que podemos cifrar en torno a algo más del triple (el valor 4,00 indicaría el punto medio entre el doble y el cuádruple). Este resultado no es ninguna sorpresa, ni es especialmente preocupante, ya que los profesores somos conscientes del gran desequilibrio existente entre ambas asignaturas, la primera con un contenido más teórico e impartida con una metodología muy clásica, y la segunda con una fuerte carga práctica, que de alguna manera se compensan. Sí resulta preocupante, en cambio, el resultado de la comparación de "Ingeniería del Software II" con el resto de asignaturas troncales de cuarto curso: los alumnos consideraron que habían dedicado bastante más esfuerzo a esta asignatura que a las demás, entre el doble y el triple, cuando lo razonable hubiera sido un esfuerzo semejante (representado por el valor 3,00). Este resultado indica, salvando incluso posibles exageraciones por parte de los alumnos, que la práctica requirió un trabajo excesivo por parte de los alumnos, a pesar del empeño de los profesores por proponer un enunciado de la práctica que requiriese tan sólo un esfuerzo moderado. Por otra parte, según los datos obtenidos mediante la Encuesta de Evaluación de la Docencia, los alumnos habían dedicado en general unas 4 horas a las demás asignaturas del curso, excluidas las clases, y

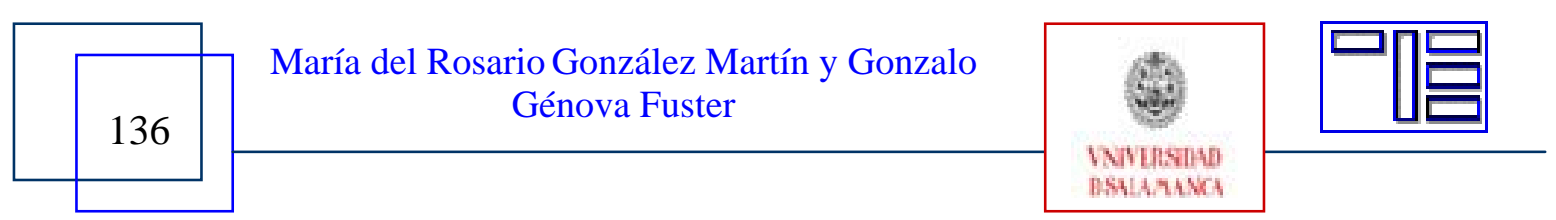


unas 8 horas a esta asignatura, es decir, más bien el doble que el triple, pero aun así resulta excesivo.

\subsection{Otros temas deseados en el temario de la asignatura}

Al ser una pregunta de respuesta libre, no podemos hacer más que agrupar las respuestas semejantes para detectar tendencias generales. En cada agrupación se indica entre paréntesis el número de respuestas más o menos semejantes obtenidas. Del mismo modo se hará en los dos apartados siguientes.

El tema que los alumnos más echan en falta es el "paso del diseño a la implementación” (7). Este deseo contrasta, por otra parte, con la estimación de los propios alumnos de haber dedicado un esfuerzo excesivo a la práctica: si además de analizarla y diseñarla, tuvieran que implementarla, el esfuerzo requerido se multiplicaría. Además, aunque es comprensible el deseo de los alumnos de ver materializado su proyecto en "algo que funcione”, ceder a este deseo significaría renunciar al objetivo principal de la asignatura, que es centrarse en el análisis y diseño de aplicaciones.

Los alumnos hubieran deseado profundizar en algunos temas de diseño, lo que concuerda que en general esta parte de la asignatura les resultó más interesante: "profundizar en diseño arquitectónico y diseño detallado” (4), “ver más patrones” (3), “explicar mejor los patrones de diseño y los estilos arquitectónicos” (2), "ver los diagramas de componentes” (2), “implementación de patrones” (1). Es una sugerencia que hay que tener en cuenta.

En relación con los estándares, se sugiere incluir una "introducción a Métrica y otros estándares” (2), “explicar el estándar de la ESA más a fondo” (2), “aclarar la relación entre el estándar ESA y la orientación a objetos” (1), y “más desarrollo con UML” (1). En cuanto a la metodología, varias respuestas van encaminadas a "ver algún ejemplo" (3), "ver algún ejemplo real en una empresa” (1), y "trabajar con clientes reales” (1). Otros temas heterogéneos que se sugieren son la "planificación de proyectos” (1), las “pruebas” (1), el "análisis de riesgos" (1), y la "realización de prototipos” (1). Todos estos temas son sin duda muy interesantes, pero en general son más propios de otras asignaturas del plan de estudios (singularmente "Ingeniería del Software 3" y "Sistemas Informáticos”, ambas de quinto curso).

\subsection{Puntos más importantes aprendidos}

Un número muy importante de alumnos señala que ha aprendido a "aplicar estándares de ingeniería” (23), y la “metodología de la ESA para documentar proyectos” (8). En cuanto a otras capacidades generales, muchos indican con satisfacción que han aprendido a "trabajar en equipo" o "trabajar en equipos grandes, de más de dos personas" (19). También son numerosas las respuestas que apuntan a la eficacia de las "revisiones cruzadas, con las que se aprende tanto de los errores propios como de los ajenos" (11), y la necesidad de pasar el mal trago de las "exposiciones orales” (7). Otros dicen "haber asimilado bien los conceptos teóricos gracias a la práctica” (3), “haber aprendido

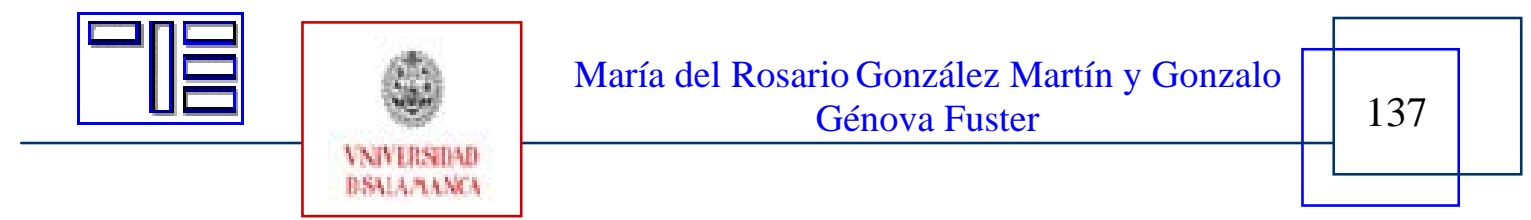


a escribir y corregir lo escrito antes de presentarlo" (1), y que una "práctica con enunciado abierto" les ha ayudado a plantear y resolver un problema complejo (1), debiendo ellos mismos "establecer los criterios de corrección" (1).

El planteamiento de la práctica les ha ayudado a "entender un proyecto con sus pasos reales” (13), “enfrentarse a un proyecto grande” (9), "valorar la planificación del proyecto” (6), especialmente las “entregas semanales” (2), y más de uno señala con sorpresa que "el proyecto parecía más sencillo al empezar" (2), y han descubierto la "importancia de la trazabilidad" (2).

Un grupo de respuestas se centran en la primera parte del temario: han aprendido a "obtener los requisitos” (7), “organizar los requisitos” (5), "valorar la ingeniería de requisitos” (3), "valorar el análisis de requisitos” (3), y la “diferencia precisa entre los requisitos de usuario y requisitos del software" (1).

Otras respuestas tienen que ver más bien con la segunda parte del temario: “diseño arquitectónico” (9), “patrones de diseño” (7), “diseño detallado” (3), “valorar los patrones y la reutilización” (1), y los “marcos” (1).

Finalmente, algunas respuestas heterogéneas: “diseñar sistemas” (1), "representación gráfica y textual” (1), “pensar en la solución” (1), y “debates de la asignatura” (1). En general, sin duda resulta muy satisfactorio comprobar la opinión de los mismos alumnos sobre lo mucho que han aprendido en la asignatura, especialmente en relación con los objetivos del presente Proyecto de Innovación Docente.

\subsection{Otras sugerencias}

La sugerencias más numerosas van en la línea de "simplificar práctica, ya que ha requerido un trabajo excesivo" (13), "flexibilizar los plazos" (2), "restringir los apartados del estándar ESA que es necesario cumplimentar” (1), “dedicar horas lectivas al desarrollo de la práctica” (1), y “es demasiado trabajo leer los estándares” (1). No obstante, alguno señala que "la práctica no se hizo pesada gracias a trabajo en equipo" (1). Como se trataba de una práctica de enunciado muy abierto, los profesores insistimos muchas veces durante el curso en que no debían complicar ellos mismos la práctica, añadiendo requisitos innecesarios que no harían más que aumentarles el trabajo: alguno ha considerado "indignante culpar a los alumnos de que la práctica haya resultado excesivamente complicada” (1). En todo caso, es cierto que la práctica ha resultado excesiva, y será necesario tomar medidas eficaces para que esto no vuelva a ocurrir, a pesar de que algunos alumnos están conformes con el desarrollo de la asignatura y recomiendan "mantener este formato" (2).

En otro grupo numeroso de sugerencias los alumnos piden "más apoyo de los profesores” (13) para orientar el desarrollo de la práctica, especialmente durante las sesiones de revisión, e incluso aportando "revisiones escritas por los profesores" (1). En principio, el papel de los profesores en las sesiones de revisión estaba planteado como relativamente pasivo, más moderador del debate que otra cosa, para que los alumnos se dieran cuenta que no podían eludir la responsabilidad de la revisión. No obstante, parece claro que será necesario adoptar un papel más activo en el futuro.

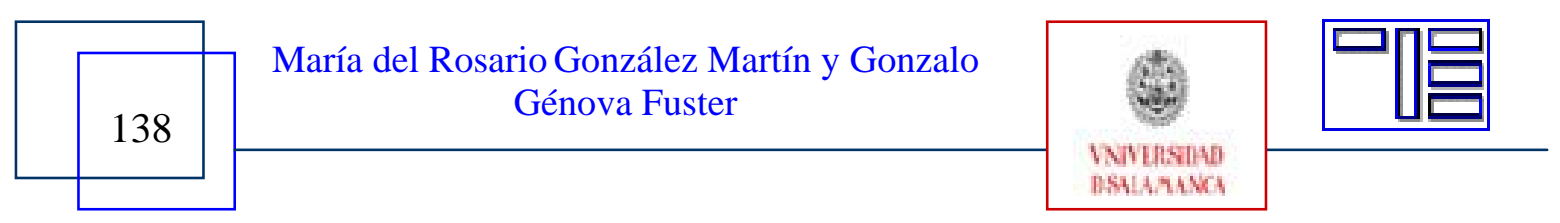


Varios alumnos sugieren "que el examen de test no sea eliminatorio" (4), o "que cuente más en la nota final” (1). También se sugiere "que las preguntas que todos responden acertadamente no sean eliminadas para la calificación” (1); esta regla se propuso con la intención de disuadir a los alumnos de difundir a sus compañeros su propia propuesta de preguntas, pero finalmente no fue aplicada, entre otros motivos por que se habrían eliminado prácticamente todas las preguntas, dado el elevadísimo porcentaje de preguntas acertadas en el test. Realmente, este conjunto de medidas, orientadas a potenciar el valor de la teoría y del examen en la nota final, no parecen eficaces, de modo que habrá que hacer caso de las sugerencias recibidas.

Dos importantes sugerencias señalan que algunos temas ya se han visto en asignaturas troncales del plan de estudios: "patrones de diseño en Técnicas de Desarrollo de Programas” (1) y “obtención de requisitos en Interfaces de Usuario” (1). La primera ya era conocida, la segunda habrá que investigarla más a fondo, y en todo caso es necesaria una mejor coordinación con esas asignaturas.

Finalmente, otras sugerencias heterogéneas: “excesivo número de correos a los profesores” (1), "no exigir formato Word para los documentos” (1), "poner los apuntes a disposición de los alumnos antes de la clase”, y (1) "no es bueno que los compañeros evalúen” (1). Respecto a esta última sugerencia, en realidad no se les ha pedido que evalúen, sino que revisen y corrijan el trabajo de sus compañeros, sin calificarlo. La mayoría lo han entendido perfectamente.

\section{5.- CONCLUSIONES Y PROPUESTAS DE APLICACIÓN}

Todo el equipo de profesores participantes quedó muy satisfecho de los resultados obtenidos en este proyecto. Los resultados percibidos son muy buenos, tanto por la calidad de las prácticas entregadas como por el nivel de conocimientos y habilidades adquiridos por los alumnos. Esto se refleja naturalmente en las calificaciones obtenidas, con un 100\% de aprobados sobre presentados (tan sólo 7 alumnos, aproximadamente el 5\%, no realizaron la práctica), y con una calificación promedio de 8,7.

El inconveniente mayor fue el excesivo volumen de trabajo que los alumnos dedicaron a la práctica, a pesar de nuestros esfuerzos por evitarlo, proponiendo un enunciado que pensábamos era realmente sencillo, y animando a los propios alumnos a limitar el tamaño de sus trabajos.

En el curso 2005-2006 solucionamos este problema dividiendo la asignatura en dos partes. La primera parte (ingeniería de requisitos y análisis) en el primer cuatrimestre, en lugar del antiguo programa de Ingeniería del Software I, que por diversos motivos se había quedado obsoleto. La segunda parte (diseño arquitectónico y diseño detallado), en estrecha continuidad con la primera, en el segundo cuatrimestre, en Ingeniería del Software II. Se incrementó ligeramente el número de clases teóricas en total, pero sin llegar a duplicarlas, para aproximarnos al modelo de Bolonia, que propugna menos lecciones magistrales y más trabajo de los alumnos. Desde entonces se ha seguido fundamentalmente la misma metodología en ambas asignaturas, añadiendo sesiones de seguimiento personalizado del profesor a cada uno de los equipos de trabajo durante las horas lectivas, respondiendo así a una de las demandas más apremiantes de los alumnos.

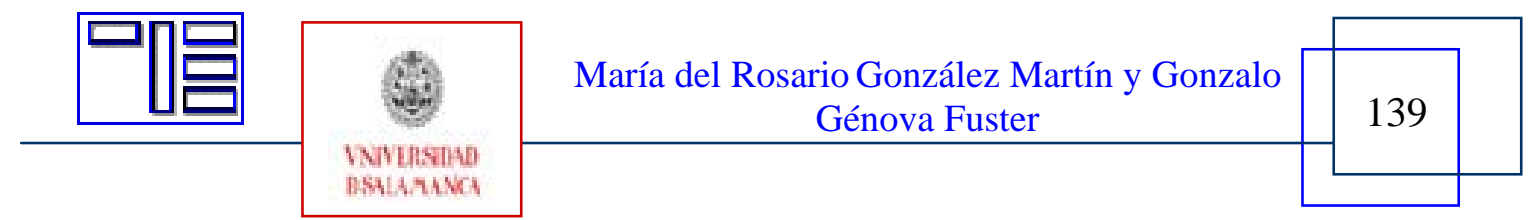


El resultado ha sido pasar de un promedio de 329 horas/equipo en el curso 2004-2005 (proyecto original) a las cifras de 283 y 234 horas/equipo respectivamente en el primer y segundo cuatrimestre del curso 2005-2006, y 231-220 horas/equipo en el curso 20062007, que son bastante más razonables.

Creemos que la conclusión principal de la experiencia de innovación puesta en marcha es la posibilidad de favorecer, gracias a un cambio metodológico del equipo de profesores, una actitud activa del alumno en su proceso de aprendizaje de una manera grupal. Pensamos que con nuestra propuesta se desarrollan competencias propias de las asignaturas concretas impartidas, así como competencias transversales requeridas por nuestra sociedad y urgidas por el Proceso de Bolonia. Entre otras competencias, hemos fomentado:

- $\quad$ La adaptación a estándares de calidad.

- $\quad$ El trabajo en equipo.

- $\quad$ El desarrollo de estrategias de comunicación: para el trabajo en equipo, para la exposición de propuestas, para la expresión de valoraciones de colegas de manera adecuada, y para la aceptación de las correcciones.

- $\quad$ La adquisición de criterios de valoración, la redacción de pruebas de evaluación y el incremento de la capacidad crítica.

Pensamos que el protagonismo del aprendizaje se ha desplazado verdaderamente al trabajo del alumno o del grupo de alumnos y que ha habido una mejora de las calificaciones sin renunciar a los objetivos propios de la asignatura, aunque sí incorporando otros nuevos que creemos que deben ser irrenunciables en este momento (competencias de comunicación y de valoración). El alumno se ha involucrado directa y personalmente en el proceso de aprendizaje, ayudando a sus compañeros a corregir sus errores, y aprendiendo a su vez de los errores de sus compañeros, sin renunciar a sistemas de control por parte del profesor que garanticen la veracidad y aseguren la adquisición real de competencias.

Creemos que ha sido una experiencia muy positiva, y seguiremos trabajando en ella llevándola a otros ámbitos de conocimiento, ya que la esencia de la innovación de este proyecto (el trabajo en equipo, las revisiones cruzadas y la propuesta de preguntas para examen que reflejen aspectos centrales de la asignatura) es una metodología fácilmente trasladable a otras áreas.

\section{6.- REFERENCIAS}

CONSEJO DE COORDINACIÓN UNIVERSITARIA (2006) Propuesta para la renovación de las metodologías educativas de la Universidad. M.E.C. Madrid.

MICHAVILA, F.; MARTÍNEZ, J. (2004) (eds.). La profesión del profesor de universidad. Universidad Politécnica de Madrid, Cátedra UNESCO de Gestión y Política Universitaria, Madrid.

MICHAVILA, F.; GARCÍA DELGADO, J. (2003) (eds.) La tutoría y los nuevos modos de aprendizaje en la universidad. Universidad Politécnica de Madrid, Cátedra UNESCO de Gestión y Política Universitaria, Madrid.

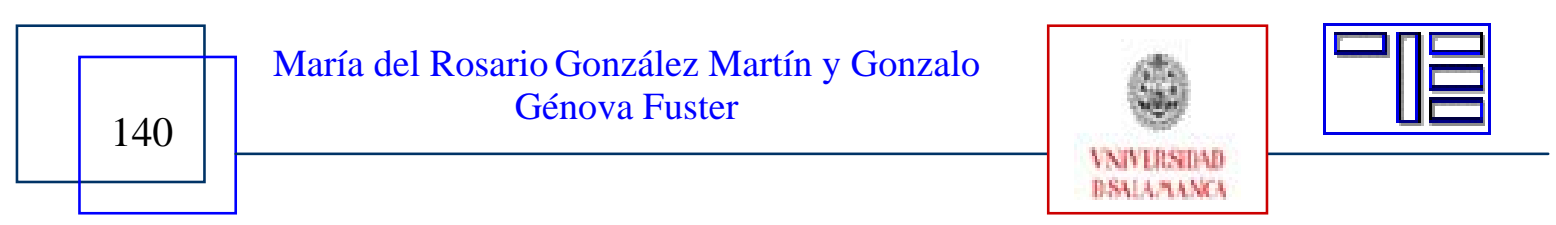


MICHAVILA, F.; MARTÍNEZ, J. (2002) (eds.). El carácter transversal de la educación universitaria. Universidad Politécnica de Madrid, Cátedra UNESCO de Gestión y Política Universitaria, Madrid.

COMUNICADO DE LA CONFERENCIA DE MINISTROS DE Bergen (2005) www.institucional.us.es/eees/formacion/Bergen_Comunicado-esp.pdf DECLARACIÓNDE GLASGOW (2005) www.institucional.us.es/eees/formacion/Glasgow_Declaracion_esp.pdf COMUNICADO DE LA CONFERENCIA DE MINISTROS DE BERLÍN (2003) www.institucional.us.es/eees/formacion/comunicado_Berlin_2003_esp.pdf DECLARACIÓN DE GRAZ. (2003) Hasta el 2010 y más allá www.crue.org/espaeuro/encuentros/EEES_DeclFINAL_Graz.pdf COMUNICADO DE PRAGA (2001) Hacia el Área de la Educación Superior Europea www.ugr.es/ fbd/ects/Nueva\%20carpeta/declaracionpraga.pdf INFORME DE SALAMANCA (2001) www.unizar.es/eees/doc/Informe_Salamanca_2001.pdf DECLARACIÓN DE BOLONIA (1999) magno.uab.es/fas/piune/normativa/declaracion_bolonia.pdf DECLARACIÓN LA SORBONA (1998) www.usal.es/webusal/Novedades/noticias/bolonia/sorbona.pdf

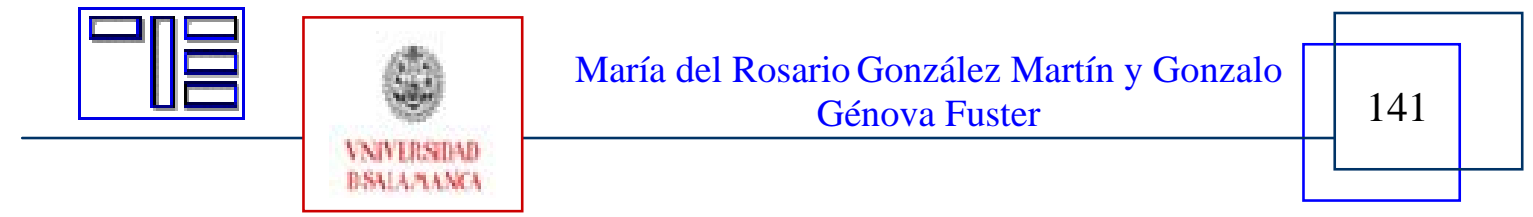

